INTRODUCTION 


\title{
Development in astronomy in Ethiopia and East-Africa through nuclear activity in galaxies
}

\author{
Mirjana Pović ${ }^{1,2}$ \\ ${ }^{1}$ Astronomy and Astrophysics Research and Develoment Division, Etiopian Space Science and \\ Technology Institute (ESSTI), Addis Ababa, Ethiopia \\ ${ }^{2}$ Instituto de Astrofísica de Andalucía (IAA-CSIC), Granada, Spain
}

\begin{abstract}
In this paper we summarise the research that is currently going on in Ethiopia and East-Africa in extragalactic astronomy and physics of active galaxies and active galactic nuclei (AGN). The study is focused on some of the still open questions such as: what are the stellar ages and populations of ultra hard X-ray detected AGN and connection between AGN and their host galaxies?, what are the properties of AGN in galaxy clusters and the role that environment has in triggering nuclear activity?, what are the morphological properties of AGN and how precisely we can deal with morphological classification of active galaxies?, what are the properties of galaxies in the green valley and the role of AGN in galaxy evolution?, and what are the properties of radioloud and radio-quiet quasars (QSO) and dichotomy between the two?. Each of these questions has been developed under one specific project that will be briefly introduced. These projects involve $6 \mathrm{PhD}$ and $3 \mathrm{MSc}$ students and collaborations between Ethiopia, Rwanda, South Africa, Uganda, Tanzania, Spain, Italy, and Chile. With all projects we aim: first, to contribute to our general knowledge about AGN, and second, to contribute to the development in astronomy and science in Ethiopia and East-Africa.
\end{abstract}

Keywords. galaxies: active; galaxies: main properties; astronomy development in Africa

\section{Introduction}

How galaxies form and evolve is still one of the fundamental questions in modern cosmology. Many properties of different types of galaxies are still fairly understood, especially when going to higher redshifts. In particular, active galaxies, having active galactic nuclei (AGN) in their centre, play an important role in understanding galaxy formation and evolution, supermassive black hole (SMBH) formation and growth, star formation (SF) in galaxies, morphological transformation and role that AGN may have in moving galaxies from late- to early-types, or how the Universe was in its early stage (e.g., Heckman \& Best 2014; Netzer 2015; Hickox \& Alexander 2018). In the following we describe briefly several projects that are related with some of the still open questions in the field of AGN, and in the same time are important for human capacity building and astronomy development in Ethiopia and East-Africa.

\section{Stellar ages and morphologies of ultra-hard AGN}

Connection between SF and AGN activity was studied widely over the past years, and shown to be very important for understanding the role of AGN in galaxy evolution (e.g., Pović et al. 2013; Shimizu et al. 2017; Masoura et al. 2018). What are the stellar ages and average stellar populations of AGN host galaxies, and if there are differences depending on AGN type, are still some of open questions. The AGN sample detected in the ultra-hard

(C) The Author(s), 2021. Published by Cambridge University Press on behalf of International Astronomical Union 
X-rays $(14-195 \mathrm{keV})$ by the Swift BAT telescope is not affected by obscuration nor it is contaminated by stellar emission, and therefore presents some of the most unbiased samples. Therefore, the Swift-BAT AGN Spectroscopic Survey (BASS†; Koss et al. 2017) gives us an unique opportunity to understand connection between AGN and their host galaxies by studying the SMBH mass and accretion rate (Koss et al. 2017; Ricci et al. 2017) in relation to stellar properties, metallicities, and morphologies of AGN hosts. It is for the first time that this kind of study will be carried out for a complete sample of ultra-hard X-ray detected AGN.

Using the optical spectra and BASS DR1 data (Koss et al. 2017) we are carrying out spectral energy distribution (SED) fitting by using the STARLIGHT code (Cid Fernandes et al. 2004), with aim to study stellar ages and populations of ultra-hard X-ray AGN. For fitting the type-2 AGN, we are following the same procedure as in (Pović et al. 2016), and testing templates with different metallicities and stellar ages Bruzual \& Charlot (2003). Using the obtained emission spectra we are measuring properties of all emission lines (integrated fluxes and equivalent widths), again using the same methodology as in (Pović et al. 2016). Metallicities will be measured using the new Bayesian-like approach of Pérez-Montero et al. (2019) that has been tested on type-2 AGN. This study will be combined by morphological analysis of the same sample. Visual multiwavelength classification in optical, radio, and X-rays was carried out (see Bilata-Woldeyes et al. 2020, poster paper in Chapter 8), where we obtained that most of ultra hard X-ray detected AGN are hosted by spirals in optical, are radio-quiet, and have compact morphologies in X-rays. We are finally planning to study in more details how/if X-ray luminosities, $\mathrm{SMBH}$ masses, and accretion rates are correlated with obtained stellar ages and populations, metallicities, and morphology, for understanding better connection between the ultra-hard AGN and their host galaxies.

This project is carried out as a collaboration between Ethiopia and Spain. Morphological analysis resulted in MSc degree of Betelehem Bilata, one of our few female students.

\section{Properties of galaxies in galaxy clusters up to $\mathrm{z} \sim 1.0$}

The study of properties of galaxies inside galaxy clusters represents one of the main steps in understanding the formation and evolution of the Universe. In particular, it is important to understand how galaxies transform inside the clusters and how they change their properties as a function of redshift and environment. The research case proposed here has been carried out at the ESSTI under the GLACE collaboration (GaLAxy Cluster Evolution survey; Sánchez-Portal et al. 2015), with general aim to study the properties and evolution of galaxies in galaxy clusters up to $\mathrm{z} \sim 1$. Our main objective is to better understand some of the still open questions such as: the role of AGN in galaxy clusters, metallicity variability, and galaxy transformation and evolution within clusters at different cosmic times.

Two GLACE clusters, RXJ1257.2+4738 at $\mathrm{z}=0.866$ and $\mathrm{ZwCl0024.0+1652}$ at $\mathrm{z}=0.395$, have been analysed using tunable filters (TF) data available from the OSIRIS instrument at the GTC 10m telescope and public data. For the RXJ1257 cluster we carried out morphological classification and analysis (Pintos-Castro et al. 2016) using the non-parametric methods based on the galSVM code (Huertas-Company et al. 2008). Regarding ZwCl0024 cluster, emission line galaxies have been previously selected and analysed in $\mathrm{H}_{\alpha}$ and [NII] lines (Sánchez-Portal et al. 2015). We carried out morphological classification of galaxies in $\mathrm{ZwCl0024}$, in a consistent way as in RXJ1257, by classifying all galaxies as early- or late-types, and obtaining the most detailed catalogue

$\dagger$ https://www.bass-survey.com/ 
up to date up to a clustercentric distance of $1 \mathrm{Mpc}$ (Beyoro-Amado et al. 2019). Data reduction and analysis of $\mathrm{H}_{\beta}$ and [OIII] lines in the $\mathrm{ZwCl0024}$ cluster has been finalised. Obtained emission line fluxes and luminosities have been tested and compared with those of $\mathrm{H}_{\alpha}$ and [NII] (Beyoro-Amado et al. 2020, in prep). We obtained pseudo-spectra of both lines and selected possible emission line galaxies after inspection of pseudo-spectra and HST/ACS images. We are studying the nuclear activity in this cluster using the BPT diagram and 4 emission lines from TF observations. Metallicity estimates will be carried out using [NII] line, SFR using $\mathrm{H}_{\alpha}$ line, and extinctions using $\mathrm{H}_{\alpha}$ and $\mathrm{H}_{\beta}$ lines. All properties including morphology will be analysed in relation to local density and clustercentric distance. Finally, we will provide a more global analysis where properties measured for RXJ1257 and ZwCl0024 clusters will be compared with those obtained for local Virgo cluster using public data. For more information about this work see Beyoro-Amado et al. (2020) paper in Chapter 4.

This project is related to $\mathrm{PhD}$ thesis of Zeleke Beyoro-Amado, and forms a part of collaboration between Ethiopia and Spain.

\section{Morphological properties of active galaxies}

Morphology is the most accessible indicator of galaxy physical structure, being crucial for understanding the formation of galaxies throughout cosmic time and for providing answers to some of still open questions mentioned above. In case of active galaxies there are still many inconsistencies between the results obtained and their interpretation regarding morphology (e.g., Pierce et al. 2007; Georgakakis et al. 2008; Gabor et al. 2009; Pović et al. 2009, 2012; Mahoro et al. 2019), and how the AGN affects morphological classification of its host galaxies (Gabor et al. 2009; Cardamone et al. 2010; Pierce et al. 2010). In particular, the interpretation of morphology still remains a problem in the framework of galaxy evolution. Since measured morphology depends strongly on image resolution, classification in deep surveys remains very difficult, especially when dealing with faint and high redshifts sources (Pović et al. 2015). Still detailed morphological analysis is missing in the field of AGN, especially at higher redshifts.

In this work we are carrying out a detailed study on how the AGN contribution affects morphological classification of active galaxies. To do so, we are applying a similar method as used in Pović et al. (2015). Using a local sample of $\sim 2000$ visually classified SDSS inactive galaxies (Nair et al. 2010) we simulated their images by adding in the centre different AGN contributions from $5 \%$ up to $75 \%$ of the total flux. By running the galSVM code (Huertas-Company et al. 2008), we are measuring six commonly used morphological parameters and are evaluating how well the morphological classification of active galaxies can be determined at $\mathrm{z} \sim 0$ and at higher redshift in COSMOS conditions (Getachew et al. 2020a,b, in prep.). Secondly, we are planning to estimate the possible AGN contribution of COSMOS galaxies by using GALFIT code (Peng et al. 2002). Finally, taking into account the previous results we will re-classify active galaxies in the COSMOS field and understand better how they evolved across cosmic time. For more information see Getachew-Woreta et al. (2020) paper in Chapter 4.

This project is related to PhD thesis of Tilahun Getachew-Woreta, and is carried out between Ethiopia and Spain.

\section{Properties of green valley galaxies and role of AGN in galaxy evolution}

On the colour-magnitude or colour-stellar mass diagrams green valley $(\mathrm{GV})$ galaxies are located between the 'red sequence' and 'blue cloud' which are mainly populated by early- and late-type galaxies, respectively (e.g., Pović et al. 2013; Schawinski et al. 2014; Salim 2014; Bremer et al. 2018). Different studies suggested that morphological 
transformation of galaxies happens in the GV during different timescales (Schawinski et al. 2014; Smethurst et al. 2015; Trayford et al. 2016; Bremer et al. 2018), being also dependent on morphology (Nogueira-Cavalcante et al. 2018). Study of GV galaxies is therefore crucial for understanding the process of SF quenching, how galaxies transform from late- to early-types, and what is the role of AGN in morphological transformation of galaxies and their evolution.

To understand better the properties of GV sources and connection between inactive and active galaxies we selected a large sample of sources and studied their properties such as SFRs, stellar masses, sizes, morphologies, stellar populations, and ages. We are carrying out this study at low, intermediate, and higher redshifts using public SDSS, GAMA, and COSMOS data, respectively. In addition, for a smaller number of sources we are using our own spectroscopic data from SALT telescope. We measured SFRs in COSMOS through the FIR Herschel/PACS data and SED fitting, and we observed the location of active and inactive galaxies on the main-sequence (MS) of SF. We found that most of our GV X-ray detected AGN with far-IR emission have SFRs higher than the ones of inactive galaxies at fixed stellar mass ranges. Therefore, they do not show signs of SF quenching, as shown in most of previous optical studies (e.g., Nandra et al. 2007; Pović et al. 2012; Leslie et al. 2016), but rather its enhancement. Our results may suggest that for X-ray detected AGN with FIR emission if there is an influence of AGN feedback on $\mathrm{SF}$ in the GV the scenario of AGN positive feedback seems to take place, rather than the negative one (Mahoro et al. 2017). In the second paper published recently, we studied morphological properties and found that a significant number of our AGN (38\%), but not the majority, are related to interactions and mergers (Mahoro et al. 2019). At low redshifts using SDSS optical and GALEX UV data we analysed six criteria commonly used for selecting GV galaxies. We observed that depending on criteria different population of galaxies are selected, bringing therefore to different GV results (Nyiransengiyumva et al. 2020 , in prep.). For understanding better the role of AGN in SF quenching in the GV, we are now measuring stellar ages, average stellar populations, and metallicities of selected active and inactive galaxies at both lower and higher redshifts. For more information see papers of Mahoro et al. (2020) and Nyiransengiyumva et al. (2020) in Chapter 4.

This project is related to $\mathrm{PhD}$ dissertations of Antoine Mahoro and Betrice Nyiransengiyumva from Rwanda, and is carried out as a collaboration between Rwanda, Ethiopia, South Africa, Uganda, and Spain.

\section{Dichotomy of radio-loud and radio-quiet quasars}

Quasars (QSOs) were discovered more than 50 years ago. Being some of the most luminous sources in the Universe they are fundamental for cosmological studies (see paper of Marziani et al. 2020, in Chapter 2). Most of the QSOs in the local universe are radioquiet (RQ). It is still under debate if there is an evidence for a continuity or physical dichotomy between RL and RQ QSOs, what is the origin of the powerful relativistic jets, and the effect that they have on the surrounding medium. Previous studies suggested the existence of two Populations (A and B) of QSOs in the 4D Eigenvector 1 (4DE1) plane defined by the FWHM of $\mathrm{H} \beta$ and the strength of the optical FeII blend at $4570 \AA$ normalised by the intensity of $\mathrm{H} \beta$ line ( $\mathrm{RFe}$ ), and the existence of 'QSO main sequence' (Sulentic et al. 2000; Shen \& Ho 2014; Marziani et al. 2018). It has been shown that RL QSOs are strongly concentrated in the Population B (Zamfir et al. 2008). In this work we are analysing the dichotomy of RL and RQ QSOs and the effect of the relativistic radio jets on the gas in the broad line emission region.

We are using our spectroscopic data from CAHA $3.5 \mathrm{~m}$ and GTC $10 \mathrm{~m}$ telescopes of $\sim 60$ RL QSOs. We want to quantify broad emission lines differences between RL and RQ sources, exploiting larger and more complete samples of QSOs with spectral coverage 
in $\mathrm{H} \beta$, FeII, MgII and CIV emission lines than it was done previously. We are using SPECFIT code to determine the main parameters of each component. This will allow us to verify whether the larger values of $\operatorname{FWHM}(\mathrm{H} \beta)$ among $\mathrm{RL}$ sources can be due to orientation, and study the wind properties affecting the profiles of the CIV and MgII lines. Currently we are analysing a sample of 12 RL QSOs using our CAHA spectra. We will focus our comparisons on RQ and RL sources that have the same mass and $\mathrm{L} / \mathrm{L}_{E d d}$ ranges. For more information see Terefe et al. (2020) paper in Chapter 8.

This project is related with $\mathrm{PhD}$ thesis of Shimeles Terefe from Ethiopia, and is carried out in collaboration between Ethiopia, Spain, and Italy.

\section{Conclusions}

This paper describes some of the main projects that are running under the extragalactic astronomy group at the ESSTI. Several other works have been conducted related with: 'Properties of inside-out assembled galaxies at $\mathrm{z}<0.1$ ' (see Zewdie et al. 2020a,b, in Chapter 8), 'Testing the alternative method to measure the accretion rate in galaxies' (Gaulle et al. 2020, in Chapter 8), and 'Characterisation of LINERs and retired galaxies at $\mathrm{z}<0.1$ ' (Mazengo et al. 2020, in Chapter 8). These three projects resulted in three MSc dissertations in Ethiopia and Tanzania. Beside contributing to our general knowledge about galaxies and AGN, all mentioned projects contributed to development in astronomy and science in Ethiopia, Rwanda, Uganda, South Africa, and Tanzania. We managed to give more visibility to astronomy in Africa, to contribute to the institutional development of ESSTI and partner institutions, and to strengthen international collaborations. We are contributing to human capacity building of our first MSc and PhD students in the field, and we are also inspiring many other young people who went through different trainings and education and outreach activities that have been organised in Ethiopia and East-Africa over the past recent years.

\section{Acknowledgements}

Support of the Ethiopian Space Science and Technology Institute (ESSTI) under the Ethiopian Ministry of Innovation and Technology (MInT), Spanish MEC under AYA2016-76682-C3-1-P and Center of Excellence Severo Ochoa award for the IAA (SEV2017-0709) are gratefully acknowledged. This proceedings paper would not be possible without the IAUS 356 support of the IAU, ESSTI, EORC, MInT, ISP, IAA-CSIC, SEA, STFC-UKRI, DARA, ESSS, EA-ROAD, AAU, and Nature Astronomy.

\section{References}

Beyoro-Amado, Z. et al. 2019, MNRAS, 485, 1528

Beyoro-Amado, Z. et al. 2020, IAUS 356 proceedings, Cambridge University Press, in press (arXiv:2004.01892)

Bilata-Woldeyes, B. et al. 2020, IAUS 356 proceedings, Cambridge University Press, in press (arXiv:2003.12416)

Bremer, M. N. et al. 2018, MNRAS, 476, 12

Bruzual, G. \& Charlot, S. 2003, MNRAS, 344, 1000

Cardamone, C. N. et al. 2010, ApJ, 721, L38

Cid Fernandes, R. et al. 2004, ApJ, 605, 105

Gabor, J. M. et al. 2009, ApJ, 691, 705

Gaulle, A. et al. 2020, IAUS 356 proceedings, Cambridge University Press, in press (arXiv:2003.13487)

Georgakakis, A. et al. 2008, MNRAS, 385, 2049

Getachew-Woreta, T. et al. 2020, IAUS 356 proceedings, Cambridge University Press, in press (arXiv:2004.02250) 
Heckman, T. M. \& Best, P. N. 2014, ARA\&A, 52, 589

Hickox, R. C. \& Alexander, D. M. 2018, ARA $\& A$, 56, 625

Huertas-Company, M. et al. 2008, A\&BA, 478, 971

Ilbert, O. et al. 2016, ApJ, 690, 1236

Koss, M. et al. 2017, ApJ, 850, 74

Leslie, S. K. et al. 2016, MNRAS, 455, 82

Mahoro, A., Pović, M., \& Nkundabakura, P. 2017, MNRAS, 471, 3226

Mahoro, A. et al. 2019, MNRAS, 485, 452

Mahoro, A. et al. 2020, IAUS 356 proceedings, Cambridge University Press, in press (arXiv:2003.12033)

Marziani, P. et al. 2018, FrASS, 5, 6

Marziani, P. et al. 2020, IAUS 356 proceedings, Cambridge University Press, in press (arXiv:2002.07219)

Masoura, V. A. et al. 2018, A\&A, 618, 31

Mazengo, D. et al. 2020, IAUS 356 proceedings, Cambridge University Press, in press

Nair, P. B. \& Abraham, R. G. 2010, ApJS, 186, 427

Nandra, K. et al. 2007, ApJ,660, 11

Netzer, H. 2015, ARA $\mathcal{S} A, 53,365$

Nogueira-Cavalcante, J. P. et al. 2018, MNRAS, 473, 1346

Nyiransengiyumva, B. et al. 2020, IAUS 356 proceedings, Cambridge University Press, in press (arXiv:2004.01104)

Peng, C. Y. et al. 2002, AJ, 124, 266

Pérez-Montero, E. et al. 2019, MNRAS, 483, 3322

Pierce, C. M. et al. 2007, ApJ, 660, L19

Pierce, C. M. et al. 2010, MNRAS, 408, 139

Pintos-Castro, I. et al. 2016, A\&SA, 592, 108

Pović, M. et al. 2009, ApJ, 706, 810

Pović, M. et al. 2012, A\&SA, 541, A118

Pović, M. et al. 2013, MNRAS, 435, 3444

Pović, M. et al. 2015, MNRAS, 453, 1644

Pović, M. et al. 2016, MNRAS, 468, 2878

Ricci, C. et al. 2017, ApJS, 233, 17

Salim, S. 2014, Serbian Astronomical Journal, 189, 1

Sánchez-Portal, M. et al. 2015, A\&SA, 578, 30

Schawinski, K. et al. 2014, MNRAS, 440, 889

Shen, Y. \& Ho, L. C. 2014, Nature, 513, 210

Shimizu, T. T. et al. 2017, MNRAS, 466, 3161

Smethurst, R. J. et al. 2015, MNRAS, 450, 435

Sulentic, J. W. et al. 2000, ApJ, 536, 5

Terefe, S. et al. 2020, IAUS 356 proceedings, Cambridge University Press, in press (arXiv:2003.12736)

Trayford, J. V. et al. 2016, MNRAS, 460, 3925

Zamfir, S., Sulentic, J. W., \& Marziani, P., 2008, MNRAS, 387, 856

Zewdie, D. et al. 2020a, IAUS 356 proceedings, Cambridge University Press, in press (arXiv:2004.00718)

Zewdie, D. et al. 2020b, MNRAS, 498, 4345 